\title{
... und erstens kommt es anders, und zweitens, als man denkt ...
}

\section{Christoph Bosshard}

Dr. med., Vizepräsident der FMH, Departementsverantwortlicher Daten, Demographie und Qualität

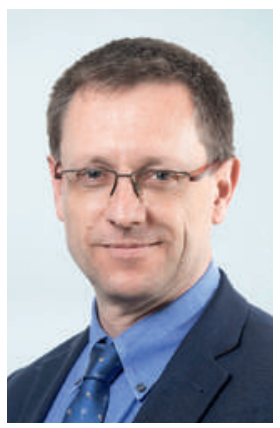

Hand aufs Herz: Wer von uns wusste schon zu Beginn seines Denkens, wo das Leben einmal hinführen würde? Es sind ja nicht nur unsere persönlichen Wünsche und Wertvorstellungen ausschlaggebend, sondern auch deren Wandel im Laufe der Zeit. Wie und wo wir unsere Prioritäten setzen und wie weit unsere Kompromissbereitschaft geht, ist sowohl individuell als auch situativ verschieden und kann sich im Laufe der Zeit verändern. Nachdem vor gut 30 Jahren Maturandinnen und Maturanden mit dem Argument der Ärzteschwemme vor einem Medizinstudium gewarnt wurden, hat sich die Situation heute ins Gegenteil verkehrt. Um den Ärztebedarf in der Schweiz zu decken, sind wir nun auf unsere Kolleginnen und Kollegen aus dem Ausland angewiesen. Oft wird in der Öffentlichkeit auch moniert, dass $\mathrm{zu}$ viele teuer ausgebildete Ärztinnen und Ärzte ihrem Beruf den Rücken kehren würden. Hinzu kommt, dass der gesellschaftliche Trend zur Teilzeitarbeit auch vor der Ärzteschaft keinen Halt macht. Um über datengestützte Diskussionsgrundlagen zur effektiven Zahl der nicht mehr kurativ tätigen Ärzte zu verfügen und um deren Gründe für die berufliche Neuorientierung zu erfahren, entschloss sich die FMH zusammen mit dem VSAO, eine Studie in Auftrag zu geben, deren Resultate in dieser Ausgabe (s. Seite 1132) vorgestellt werden.

\section{Es braucht Arbeitsmodelle mit Freiräumen für die Vereinbarkeit mit Familie und anderen individuellen Prioritäten.}

Wenn bisher stets von einer erheblichen Zahl Berufsaussteigerinnen und -aussteiger die Rede war, so lässt das Resultat doch aufhorchen. Pro Abschlussjahrgang Humanmedizin verlassen gerade etwa 10 Prozent im Laufe ihres Erwerbslebens die kurative Tätigkeit. Wir werden unser Problem des Ärztemangels also nicht mit Auflagen im Sinne einer Berufsausübungspflicht nach der Ausbildung lösen können, sondern es führt kein Weg an einer Erhöhung der Studienplätze vorbei. Die in der Botschaft zur Förderung von Bildung, Forschung und Innovation 2017-2020 zu diesem Zweck vorgesehenen Bundesmittel sind deshalb dringend nötig. Zudem wird ärztliches Wissen nicht nur am Kran- kenbett benötigt, sondern auch in der Verwaltung und in der Wirtschaft, wo die Pharmaindustrie und Medizinaltechnologie genauso wie Krankenkassen und Versicherungen auf ausgebildete Ärztinnen und Ärzte mit praktischer Erfahrung angewiesen sind. Wenn diese nicht-kurativen Tätigkeitsfelder von den 10 Prozent weggezählt werden, so wird das Potential noch kleiner, mittels Zurückholen von nicht mehr kurativ tätigen Kolleginnen und Kollegen unseren Ärztemangel zu beheben. In der Studie gibt auch nur jeder Zehnte dieser ausgestiegenen Ärztinnen und Ärzte an, sich eine Rückkehr ans Krankenbett vorstellen zu können.

Für das Problem des Ärztemangels führt kein Weg an einer Erhöhung der Medizinstudienplätze vorbei.

Vor diesem Hintergrund werden die von der FMH und vom VSAO geforderten Massnahmen noch wichtiger, damit die ärztliche Schaffenskraft zu einem möglichst hohen Anteil am Patienten bleiben kann. Angesichts der steten Zunahme der administrativen Belastung, welche die Begleitstudien zur Einführung von SwissDRG aufzeigen, wird ein klares Handlungsfeld offensichtlich. Die Lösungsideen hierzu liegen bereits seit längerem vor, aber Sekretariate kosten Geld, und Assistenzärztinnen und -ärzte waren allzu lange viel zu billige Arbeitskräfte mit unlimitierter Verfügbarkeit. Erst der Druck des Arbeitsgesetzes brachte hier eine Entwicklung in Gang, die bereits vor mehr als 10 Jahren eingesetzt hat und immer noch Verbesserungspotential aufweist. Wer sich der gesellschaftlichen Tendenz hin zur Teilzeitarbeit verschliesst, steckt den Kopf in den Sand. Es geht also darum, Arbeitsmodelle mit den notwendigen Freiräumen anzubieten, welche für die Vereinbarkeit der Arbeit am Patienten mit Familie, Partnerschaft oder anderen individuellen Prioritäten benötigt werden.

Wenn wir zukunftsfähig sein wollen, müssen wir aus den vorliegenden Resultaten lernen und die gezogenen Schlussfolgerungen umsetzen. Ich bin überzeugt, dass damit die Wertschätzung und auch die Zufriedenheit der Ärztinnen und Ärzte steigt, was sich direkt in Motivation, Patientennutzen und Effizienz niederschlagen wird. 